MATHEMATICS OF COMPUTATION

Volume 79, Number 272, October 2010, Pages 2407-2429

S $0025-5718(10) 02354-9$

Article electronically published on April 29, 2010

\title{
ON A FAMILY OF THUE EQUATIONS OF DEGREE 16
}

\author{
VOLKER ZIEGLER
}

\begin{abstract}
We consider a parameterized family of Thue equations of degree 16. By reducing this family to a system of Pell equations and linear relations, we are able to solve this family.
\end{abstract}

\section{INTRODUCTION}

It is well known that the Diophantine equation $F(X, Y)=m$, with $F \in \mathbb{Z}[X, Y]$ a homogeneous, irreducible polynomial of degree $d \geq 3$ and $m$ a nonzero integer, has finitely many solutions. This has been proved in 1909 by Axel Thue [19, and therefore equations of this type are called Thue equations. Unfortunately the proof of Thue's theorem is not constructive, i.e. we cannot find all solutions by utilizing his proof.

However, in the 1960s Baker [2] gave a method to effectively find all solutions of a given Thue equation. This method is based on Baker's theorems on linear forms in logarithms [1, 3. Baker's method was further developed by Tzanakis and de Weger [21] and by Bilu and Hanrot [6, 7]. So we have efficient algorithms to solve single Thue equations.

In 1990 Thomas [18] considered the family

$$
X^{3}-(n-1) X^{2} Y-(n+2) X Y^{2}-Y^{3}=1,
$$

where $n$ is some parameter running through all positive integers. This was the first time that a family of Thue equations was solved, where the splitting field $K$ of $F(X, 1)$ is totally real. Such families of Thue equations are usually hard to solve. However, families with real splitting field $K$ have also been solved for degree 4, 5, 6 and 8 (see e.g. [16, 10, 14, 11]). In this paper we solve a family of Thue equations of degree 16. In particular, let

$$
\alpha=\frac{(1+\sqrt{5})(\sqrt{t}+\sqrt{3}+\sqrt{2})(1+\sqrt{3})}{2} .
$$

Then we consider the Thue equation

$$
F(X, Y)=\mathrm{N}_{K / \mathbb{Q}}(X-\alpha Y)=1,
$$

where $K=\mathbb{Q}(\alpha)$ and $0<t \in \mathbb{Z}$ such that $\operatorname{deg} K=16$. This means $t$ has a prime factor $p \neq 2,3,5$ such that the highest power of $p$ which divides $t$ is not a square. We have chosen this Thue equation to demonstrate the power of our generalized

Received by the editor November 12, 2008 and, in revised form, August 13, 2009.

2010 Mathematics Subject Classification. Primary 11D59, 11Y50.

Key words and phrases. Thue equations, simultanous Pell equations, Tzanakis' method.

The author gratefully acknowledges support from the Austrian Science Fund (FWF) under project Nr. S9603.

(C)2010 American Mathematical Society Reverts to public domain 28 years from publication 
Tzanakis method. The algebraic number $\alpha$ was haphazardly taken. The only restriction was to ensure that various coefficients and constants stay adequately small. In theory we could have taken any $\alpha \in \mathbb{Q}\left(\sqrt{d_{1}}, \sqrt{d_{2}}, \sqrt{d_{3}}, \sqrt{t}\right)$ with fixed integers $d_{1}, d_{2}, d_{3}$ such that $\mathbb{Q}(\alpha)$ is of degree 16 . However, our first theorem is:

Theorem 1. Let

$$
M_{1}=\max \left\{4.793092 \cdot 10^{12} \log t(\log \log t)^{2}, 2.732786 \cdot 10^{15} \log t(\log \log t)^{2 / 3}\right\}
$$

and

$$
M=\min \left\{2.61465 \cdot 10^{18} \log t, M_{1}\right\}
$$

and assume $M<\log \eta_{t}$ or $t \leq 10^{7}$, where $\eta_{t}$ is the fundamental unit of the order $\mathbb{Z}[\sqrt{t}]$. Then Thue equation (11) has only the "trivial" solutions $X= \pm 1$ and $Y=0$.

Note that

$$
M= \begin{cases}2.7328 \cdot 10^{15} \log t(\log \log t)^{2 / 3} & \text { if } \log t<4.709 \cdot 10^{50} \\ 4.7931 \cdot 10^{12} \log t(\log \log t)^{2} & \text { if } 4.71 \cdot 10^{50}<\log t<5.78 \cdot 10^{320} \\ 2.6147 \cdot 10^{18} \log t & \text { otherwise. }\end{cases}
$$

There are a lot of papers which provide constructions of quadratic fields which have a huge fundamental unit. In particular we use a result due to Halter-Koch 9 ] in which huge fundamental units of quadratic orders are investigated. From the theorem above and the results of Halter-Koch we obtain:

Corollary 1. Let $t=12 \cdot 2^{k}+\left(1+3 \cdot 2^{k}\right)^{2}, k \geq 624761981$ and $t=84 \cdot 6^{k}+\left(5+21 \cdot 6^{k}\right)^{2}$, $k \geq 1265282$, respectively. Then Thue equation (1) has only trivial solutions.

In the second case the bound for $k$ is small enough to prove:

Theorem 2. Let $t=84 \cdot 6^{k}+\left(5+21 \cdot 6^{k}\right)^{2}$ and $k \geq 0$. Then Thue equation (11) has only trivial solutions.

In order to solve (11) we use a generalization of Tzanakis' method [20] found by the author (see section 2). In order to perform several manipulations, we have to assume $t>4000$. Therefore we compute for each $t$ all solutions to (1) using this method (section 3). Utilizing the generalized method of Tzanakis and using lower bounds for linear forms in logarithms, we find a crude upper bound for $\eta_{t}$ (section 4). An application of a method due to Baker and Davenport (see [4 or section 5) shows $t>10^{7}$. In section 6 we use this new lower bound for $t$ to sharpen our first bound for $\eta_{t}$. Let us remark that the generalized method of Tzanakis leads to an inequality of the form

$$
\left|n_{1} \log \alpha-n_{2} \log \beta+\log \gamma\right|<\epsilon^{-n_{2}},
$$

which is a motivation to apply a recent result on linear forms in three logarithms (see [8, 5] or section 7). This yields the final upper bound for $\eta_{t}$ and hence Theorem 1 .

On the other hand the Brauer-Siegel theorem (see [12, Chapter XVI]) indicates that many quadratic fields have large regulators and many families of such quadratic fields are known. In section 8 we study two families with large regulators and hence large fundamental unit $\eta>1$. These investigations will lead us to Corollary [1, Again using the method of Baker and Davenport we are able to finish the proof of Theorem 2 in section 9 .

Before we start our investigations let us note that the case $Y=0$ can be excluded, since this yields $X= \pm 1$ the trivial solutions. 


\section{Reduction to a Diophantine system}

In this section, we want to reduce Thue equation (1) to the Diophantine problem

$$
\begin{array}{r}
\left(U_{i}+A_{i} V_{i}\right)^{2}-d_{i} V_{i}^{2}=1, \\
U_{1}\left(1-\zeta_{1}-\zeta_{2}-\zeta_{3}\right)+U_{2} \zeta_{1}+U_{3} \zeta_{2}+U_{4} \zeta_{3}=U_{5}, \\
\left|\frac{U_{i}}{U_{j}}-\alpha_{i, j}\right| \leq \frac{c_{i, j}}{\left|U_{j}\right|^{2}},
\end{array}
$$

with $1 \leq i, j \leq 5$. We choose $d_{1}=2, d_{2}=3, d_{3}=t, d_{4}=10$ and $d_{5}=15$. Moreover, we write $N_{i}=\mathbb{Q}\left(\sqrt{d_{i}}\right)$ and obtain

$$
\mathrm{N}_{K / N_{i}}(X-\alpha Y)=U_{i}+\left(A_{i}+\sqrt{d_{i}}\right) V_{i},
$$

with $U_{i}, V_{i} \in \mathbb{Q}[X, Y]$ and $A_{i} \in \mathbb{Q}$. By comparing coefficients, we find

$$
\begin{aligned}
V_{1}= & -4 X Y\left(X^{6}+6 X^{5} Y+16(t-7) X^{4} Y^{2}+48(2 t-9) X^{3} Y^{3}\right. \\
& -32\left(t^{2}-3 t-7\right) X^{2} Y^{4}-24\left(7 t^{2}-30 t-1\right) X Y^{5} \\
& \left.-8(t+1)\left(t^{2}-10 t+1\right) Y^{6}\right), \\
V_{2}= & -4 Y\left(-X^{7}-3(t+1) X^{6} Y+20(t+5) X^{5} Y^{2}+4\left(11 t^{2}+18 t+59\right) X^{4} Y^{3}\right. \\
& +152\left(t^{2}-12 t-5\right) X^{3} Y^{4}-180\left(t^{3}-9 t^{2}+55 t+1\right) X^{2} Y^{5} \\
& \left.-568(t-1)\left(t^{2}-10 t+1\right) X Y^{6}+224\left(t^{2}-10 t+1\right)^{2} Y^{7}\right), \\
V_{3}= & 4 X Y\left(-X^{6}-6 X^{5} Y+8(t+8) X^{4} Y^{2}+48(3 t-1) X^{3} Y^{3}\right. \\
& \left.+16(t-4)(t+7) X^{2} Y^{4}-24\left(t^{2}+6 t+17\right) X Y^{5}-8(t-5)\left(t^{2}-10 t+1\right) Y^{6}\right), \\
V_{4}= & 4 X Y\left(-X^{6}+6 X^{5} Y+4(t+5) X^{4} Y^{2}-48 t X^{3} Y^{3}\right. \\
& \left.+8\left(t^{2}-18 t+5\right) X^{2} Y^{4}-24\left(t^{2}-10 t+1\right) X Y^{5}-8(t+1)\left(t^{2}-10 t+1\right) Y^{6}\right), \\
V_{5}= & 4 X Y\left(-X^{6}-(t-7) X^{5} Y+8(2 t+1) X^{4} Y^{2}\right. \\
& +12\left(t^{2}-6 t-3\right) X^{3} Y^{3}+16\left(2 t^{2}-21 t-1\right) X^{2} Y^{4} \\
& \left.-4(t-7)\left(t^{2}-10 t+1\right) X Y^{5}-8(t-1)\left(t^{2}-10 t+1\right) Y^{6}\right) .
\end{aligned}
$$

Now, let us write

$$
\begin{gathered}
U_{1}=X^{8}+u_{1}^{(1)} X^{7} Y+\cdots+u_{1}^{(8)} Y^{8} \\
\vdots \\
U_{5}=X^{8}+u_{5}^{(1)} X^{7} Y+\cdots+u_{5}^{(8)} Y^{8} \\
V_{1}=v_{1}^{(1)} X^{7} Y^{6}+\cdots+v_{1}^{(8)} Y^{8} \\
\vdots \\
V_{5}=v_{5}^{(1)} X^{7} Y+\cdots+v_{5}^{(8)} Y^{8}
\end{gathered}
$$


Note that the coefficients $v_{i}^{(j)}$ are known and the $u_{i}^{(j)}$ are unknown for $1 \leq i \leq 5$ and $1 \leq j \leq 8$. By (3) and the middle equation of (2) we obtain the linear system

$$
\begin{gathered}
u_{1}^{(1)}+A_{1} v_{1}^{(1)}=\tilde{c}_{1}^{(1)} \\
\vdots \\
u_{1}^{(8)}+A_{1} v_{1}^{(8)}=\tilde{c}_{1}^{(8)} \\
u_{2}^{(1)}+A_{2} v_{1}^{(1)}=\tilde{c}_{2}^{(1)} \\
\vdots \\
u_{1}^{(1)}\left(1-\zeta_{1}-\zeta_{2}-\zeta_{3}\right)+u_{2}^{(1)} \zeta_{1}+u_{3}^{(1)} \zeta_{2}+u_{4}^{(1)} \zeta_{3}+A_{5} v_{5}^{(1)}=\tilde{c}_{5}^{(1)} \\
\vdots \\
u_{1}^{(8)}\left(1-\zeta_{1}-\zeta_{2}-\zeta_{3}\right)+u_{2}^{(8)} \zeta_{1}+u_{3}^{(8)} \zeta_{2}+u_{4}^{(8)} \zeta_{3}+A_{5} v_{5}^{(8)}=\tilde{c}_{5}^{(8)},
\end{gathered}
$$

where the $\tilde{c}_{i}^{(j)}$ with $1 \leq i \leq 5$ and $1 \leq j \leq 8$ are obtained by directly computing the coefficients of the norm in equation (3). Note that this linear system is overdetermined, but we have the three parameters $\zeta_{1}, \zeta_{2}$ and $\zeta_{3}$ to play with. If we choose

$$
\begin{aligned}
\zeta_{1} & =\frac{35\left(t^{2}-42 t+65\right)}{2 t^{3}-63 t^{2}-692 t-135}, \\
\zeta_{2} & =\frac{5\left(3 t^{4}-140 t^{3}+1066 t^{2}-3292 t-325\right)}{8(t-2)\left(2 t^{3}-63 t^{2}-692 t-135\right)}, \\
\zeta_{3} & =\frac{-7\left(t^{3}-39 t^{2}-61 t+195\right)}{4\left(2 t^{3}-63 t^{2}-692 t-135\right)},
\end{aligned}
$$

then the linear system is solvable and we are able to compute the $u$ 's. In particular we find

$$
\begin{aligned}
U_{1}=X^{8} & +\left(4\left(t^{2}-10 t+1\right)\right)^{2} Y^{8}+\frac{1}{t^{4}-46 t^{3}+180 t^{2}-722 t+2315} \\
\times & \left(-\left(12 t^{4}-536 t^{3}+1792 t^{2}-4008 t+14260\right) X^{7} Y\right. \\
- & \left(24 t^{5}-1072 t^{4}+2944 t^{3}-13776 t^{2}+60392 t-7040\right) X^{6} Y^{2} \\
+ & \left(416 t^{4}-23232 t^{3}+5248 t^{2}+252608 t+41440\right) X^{5} Y^{3} \\
+ & \left(92 t^{6}-4608 t^{5}+34748 t^{4}-203552 t^{3}+315540 t^{2}+685856 t\right. \\
& -219820) X^{4} Y^{4} \\
- & \left(448 t^{5}-29504 t^{4}-69760 t^{3}+345984 t^{2}+997056 t+82880\right) X^{3} Y^{5} \\
- & \left(96 t^{7}-5248 t^{6}+57824 t^{5}-373760 t^{4}+663072 t^{3}+130688 t^{2}\right. \\
& +1767328 t-28160) X^{2} Y^{6} \\
- & \left(96 t^{7}-5600 t^{6}+71008 t^{5}-370656 t^{4}+1595936 t^{3}-3859616 t^{2}\right. \\
& \left.+1503264 t-114080) X Y^{7}\right),
\end{aligned}
$$




$$
\begin{aligned}
& U_{2}=X^{8}+\frac{1}{7}\left(-36 X^{7} Y-24(t+1) X^{6} Y^{2}+48(t+5) X^{5} Y^{3}\right. \\
& +\left(44 t^{2}+72 t+236\right) X^{4} Y^{4}+\left(96 t^{2}-1152 t-480\right) X^{5} Y^{3} \\
& +\left(-96 t^{3}+864 t^{2}-5280 t-96\right) X^{2} Y^{6} \\
& +\left(-288 t^{3}+3168 t^{2}-3168 t+288\right) X^{7} Y \\
& \left.+\left(112 t^{4}-2240 t^{3}+11424 t^{2}-2240 t+112\right) Y^{8}\right), \\
& U_{3}=X^{8}+\frac{1}{t^{4}-140 t^{3}+1066 t^{2}-3292 t-325} \\
& \times\left(\left(-12 t^{4}+1128 t^{3}-11184 t^{2}+29976 t+3900\right) X^{7} Y\right. \\
& +\left(-48 t^{5}+2240 t^{4}-13648 t^{3}+11152 t^{2}+106048 t+15600\right) X^{6} Y^{2} \\
& +\left(384 t^{5}-23136 t^{4}+186816 t^{3}-351744 t^{2}-400704 t-93600\right) X^{5} Y^{3} \\
& +\left(372 t^{6}-18776 t^{5}+118188 t^{4}+32304 t^{3}-629236 t^{2}-1297240 t\right. \\
& -61100) X^{4} Y^{4} \\
& +\left(768 t^{6}-52416 t^{5}+714816 t^{4}-3606912 t^{3}+7391616 t^{2}-1685952 t\right. \\
& +187200) X^{3} Y^{5} \\
& +\left(-192 t^{7}+10880 t^{6}-153600 t^{5}+1256896 t^{4}-6220480 t^{3}+13049856 t^{2}\right. \\
& -1861760 t+62400) X^{2} Y^{6} \\
& +\left(-96 t^{7}+9312 t^{6}-164448 t^{5}+1094496 t^{4}-2762784 t^{3}-716256 t^{2}\right. \\
& +408288 t-31200) X Y^{7} \\
& +\left(48 t^{8}-3200 t^{7}+66752 t^{6}-623232 t^{5}+2832800 t^{4}-5611904 t^{3}\right. \\
& \left.\left.+540096 t^{2}+51328 t-5200\right) Y^{8}\right), \\
& U_{4}=X^{8}+\frac{1}{t^{3}-39 t^{2}-61 t+195} \\
& \times\left(\left(-12 t^{3}+468 t^{2}+4572 t-2340\right) X^{7} Y\right. \\
& +\left(-24 t^{4}+968 t^{3}+216 t^{2}-29672 t+6240\right) X^{6} Y^{2} \\
& +\left(288 t^{3}-26592 t^{2}-94368 t+56160\right) X^{5} Y^{3} \\
& +\left(92 t^{5}-3580 t^{4}-5416 t^{3}+181960 t^{2}-29428 t+99060\right) X^{4} Y^{4} \\
& +\left(-192 t^{4}-23808 t^{3}+587136 t^{2}-155904 t-112320\right) \\
& +\left(-96 t^{6}+4832 t^{5}-41024 t^{4}+180672 t^{3}-443104 t^{2}-783008 t\right. \\
& +24960) X^{6} Y^{2} \\
& +\left(-96 t^{6}+4800 t^{5}-5664 t^{4}-318336 t^{3}-9888 t^{2}-181056 t+18720\right) X^{7} Y \\
& +\left(16 t^{7}-944 t^{6}+13136 t^{5}-41328 t^{4}-149456 t^{3}+337136 t^{2}\right. \\
& \left.-63376 t+3120) Y^{8}\right)
\end{aligned}
$$




$$
\begin{aligned}
U_{5}=X^{8} & +\frac{1}{2 t^{3}-63 t^{2}-692 t-135} \\
\times & \left(\left(-9 t^{3}+651 t^{2}-7491 t+4545\right) X^{7} Y\right. \\
+ & \left(-33 t^{4}+1446 t^{3}-2988 t^{2}+66906 t-30195\right) X^{6} Y^{2} \\
+ & \left(240 t^{4}-13464 t^{3}+84456 t^{2}+13944 t-29880\right) X^{5} Y^{3} \\
+ & \left(244 t^{5}-13080 t^{4}+100488 t^{3}-479760 t^{2}-99676 t+128520\right) X^{4} Y^{4} \\
+ & \left(480 t^{5}-31824 t^{4}+452544 t^{3}-2006688 t^{2}+1470432 t+59760\right) X^{3} Y^{5} \\
+ & \left(-132 t^{6}+7104 t^{5}-76068 t^{4}+586464 t^{3}-683148 t^{2}\right. \\
& +1890144 t-120780) X^{2} Y^{6} \\
+ & \left(-72 t^{6}+6000 t^{5}-118008 t^{4}+752928 t^{3}-1064376 t^{2}\right. \\
& +459888 t-36360) X Y^{7} \\
+ & \left(32 t^{7}-1648 t^{6}+12352 t^{5}+115824 t^{4}-1065952 t^{3}+112 t^{2}\right. \\
& \left.+32128 t-2160) Y^{8}\right),
\end{aligned}
$$

and, moreover, we have

$$
\begin{gathered}
A_{1}=-\frac{4\left(t^{3}-23 t^{2}+291 t-845\right)}{t^{4}-46 t^{3}+180 t^{2}-722 t+2315}, \quad A_{2}=\frac{12}{7}, \\
A_{3}=\frac{6\left(t^{4}-23 t^{3}+67 t^{2}-397 t\right)}{3 t^{4}-140 t^{3}+1066 t^{2}-3292 t-325}, \quad A_{4}=\frac{960 t}{t^{3}-39 t^{2}-61 t+195}, \\
A_{5}=\frac{15\left(t^{3}-7 t^{2}-1053 t+195\right)}{4\left(2 t^{3}-62 t^{2}-692 t-135\right)} .
\end{gathered}
$$

Therefore we have reduced Thue equation (11) to the first two equations of system (2).

Let us choose the index $j$ such that

$$
\left|X-\alpha_{j} Y\right|=\min _{i}\left|X-\alpha_{i} Y\right|
$$

where $\alpha_{1}:=\alpha, \ldots, \alpha_{16}$ are the conjugates of $\alpha$. We assume $j=1$ for our further considerations, since for $t>4000$ (see next section) the other cases run analogously and only absolute constants may change. Furthermore, we write $f(X):=F(X, 1)$. Then we know by the classical theory of Thue equations that

$$
X=\alpha Y+\theta \frac{2^{15}}{\left|Y^{15} f^{\prime}(\alpha)\right|}=\alpha Y+\theta \frac{1.399332}{\mid Y^{15} t^{15 / 2}},
$$

where $|\theta| \leq 1$. If we insert this into the expressions for the $U$ 's we get

$$
U_{i}=Y^{8} b_{i}+\theta \frac{R_{i}}{|Y|^{8}}
$$

where $b_{i}$ is an explicit computable algebraic number, $|\theta| \leq 1$ and $R_{i}$ is effective computable and depends only on $t$. From the relation above we obtain

$$
\left|\frac{U_{i}}{U_{j}}-\alpha_{i, j}\right| \leq \frac{R_{i, j}}{|Y|^{16}}=\frac{c_{i, j}}{\left|U_{j}\right|^{2}}
$$

where $R_{i, j}$ and $c_{i, j}$ are again effective computable and $\alpha_{i, j}=\frac{b_{i}}{b_{j}}$. Hence we have shown how to reduce Thue equation (1) to Diophantine system (2). 


\section{SMALL $t$ : I}

In this section, we roughly show how to solve Diophantine system (2) and in particular we describe how to solve the case $t \leq 4000$. Note that the first equations in (2) are Pell equations. Hence we have

$$
\begin{aligned}
U_{i}+A_{i} V_{i} & = \pm \frac{\epsilon_{i}^{n_{i}}+\epsilon_{i}^{-n_{i}}}{2} \\
V_{i} & = \pm \frac{\epsilon_{i}^{n_{i}}-\epsilon_{i}^{-n_{i}}}{2 \sqrt{d_{i}}}
\end{aligned}
$$

with $\epsilon_{1}=3+2 \sqrt{2}, \epsilon_{2}=2+\sqrt{3}, \epsilon_{3}=\eta_{t}=\eta, \epsilon_{4}=19-6 \sqrt{10}$ and $\epsilon_{5}=4+\sqrt{15}$ and with exponents $n_{i} \geq 0$. Hence

$$
U_{i}=\beta_{i} \epsilon_{i}^{n_{i}}+\bar{\beta}_{i} \epsilon_{i}^{n_{i}}
$$

Note that the $\beta$ 's are effective computable for each given $t$. Hence we obtain from the last two lines of system (2) the inequality

$$
\left|\beta_{1} \epsilon_{1}^{n_{1}}\left(\zeta_{1}+\zeta_{2}+\zeta_{3}-1\right)+\beta_{2} \epsilon_{2}^{n_{2}}\left(\zeta_{1}+\alpha_{2,3} \zeta_{2}+\alpha_{2,4} \zeta_{3}-\alpha_{2,5}\right)\right| \leq c \epsilon_{2}^{-n_{2}}
$$

with an effective computable constant $c$ depending only on $t$. Note that we also have $n_{1} c_{1}<n_{2}<n_{2} c_{2}$ for some computable constants $c_{1}$ and $c_{2}$. Let us put

$$
\gamma=\frac{\beta_{1}\left(1-\zeta_{1}-\zeta_{2}-\zeta_{3}\right)}{\beta_{2}\left(\zeta_{1}+\alpha_{2,3} \zeta_{2}+\alpha_{2,4} \zeta_{3}-\alpha_{2,5}\right)}
$$

Then we have

$$
\left|\frac{\epsilon_{1}^{n_{1}} \gamma}{\epsilon_{1}^{n_{2}}}-1\right| \leq \frac{c}{2} \epsilon_{2}^{-2 n_{2}}
$$

and taking logarithms and observing $|\log x|<2|1-x|$ for $|1-x|<1 / 3$ we obtain

$$
|\Lambda|:=\left|n_{1} \log \epsilon_{1}-n_{2} \log \epsilon_{2}+\log \gamma\right|<c \epsilon_{2}^{-2 n_{2}}
$$

with a new effective computable constant $c$. Using lower bounds for linear forms in logarithms, in particular using a result of Matveev [15] (see also the next section), we obtain

$$
\log |\Lambda|>-c^{\prime} \log n_{2}
$$

hence $n_{2}<N_{0}$, where again $N_{0}$ is an effective computable constant. Using a method due to Baker and Davenport [4] (see also Section 5) or a method based on the LLL-algorithm (see [17, section VI.3]) we can reduce the usually huge first bound $N_{0}$ to a suitable smaller bound $N$, i.e. we can find suitable small upper bounds for $\left|U_{2}\right|$ and also $|Y|$. By the classical theory of Thue equations it is known that a solution $(X, Y)$ yields a convergent $X / Y$ to $\alpha$, provided $Y$ is not too small. Therefore it is possible to find all solutions to Thue equation (1) in theory and also in practice for $t \leq 4000$. The author implemented the idea presented in this section using Matveev's theorem [15] and the upper bound reduction based on the LLL-algorithm in Mathematica. The program was running 2 days, 10 hours and 3 minutes on a common work station and found no nontrivial solutions for $t \leq 4000$. Note that the implementation is rather crude and improvements of the implementation may reduce the running time to less than one day. For details on how to compute the bounds occurring in this section, see below. 


\section{A FIRST BOUnd}

In this section, we assume $t>4000$. Note that for different choices of $j$ (the last paragraph of section 2) we obtain different Diophantine systems (2). Since the computations for different $j$ 's are nearly the same, we give details only for $j=1$. Note that in this section the constants $c_{1}, \ldots$ are only depending on $t$ and are effective computable.

First, we want to compute the $\beta$ 's occurring in (9). By utilizing (3) and (6) we find

$$
\begin{aligned}
U_{1}+A_{1} V_{1}>323545 \cdot t^{7 / 2}, & V_{1}<-228781 \cdot t^{7 / 2}, \\
U_{2}+A_{2} V_{2}>61506 \cdot t^{4}, & V_{2}<-35510 t^{4}, \\
U_{3}+A_{3} V_{3}>72541 \cdot t^{4}, & V_{3}<-72541 t^{7 / 2} .
\end{aligned}
$$

In other cases the $V$ 's may be positive, and we obtain other values for the $\beta$ 's (see Lemma 1 below):

Lemma 1. Assume $t>4000$. Then we have

$$
U_{i}= \pm\left(\beta_{i} \epsilon_{i}^{n_{i}}+\bar{\beta}_{i} \epsilon_{i}^{-n_{i}}\right)
$$

with $i=1,2,3$ and

$$
\begin{gathered}
\beta_{1}=\frac{1}{2} \pm \frac{\sqrt{2}\left(t^{3}-23 t^{2}+291 t-845\right)}{t^{4}-46 t^{3}+180 t^{2}-722 t+2315}, \quad \beta_{2}=\frac{1}{2} \pm \frac{2 \sqrt{3}}{7}, \\
\beta_{3}=\frac{1}{2} \pm \frac{\sqrt{t}\left(3 t^{3}-69 t^{2}+201 t-1191\right)}{3 t^{4}-140 t^{3}+1066 t^{2}-3292 t-325}
\end{gathered}
$$

and $\bar{\beta}_{i}$ are the conjugates of $\beta_{i}$ with $i=1,2,3$. The $+/-$ signs hold according to Table 1 .

TABLE 1 . The signs that hold for $\beta_{i}$ in case $j$.

\begin{tabular}{|c||c|c|c|c|c|c|c|c|c|c|c|c|c|c|c|c|}
\hline$i \backslash j$ & 1 & 2 & 3 & 4 & 5 & 6 & 7 & 8 & 9 & 10 & 11 & 12 & 13 & 14 & 15 & 16 \\
\hline \hline 1 & - & + & - & - & - & + & + & + & - & - & - & + & + & + & - & + \\
\hline 2 & + & + & - & + & + & - & + & + & - & - & + & - & - & + & - & - \\
\hline 3 & + & + & + & + & - & + & + & - & + & - & - & + & - & - & - & - \\
\hline
\end{tabular}

Now let us consider equation (77). As mentioned above it is possible to compute the $b_{i}$ 's explicitly. Doing so and assuming $t>4000$ and $j=1$, we obtain:

\section{Lemma 2.}

$$
\begin{gathered}
\left|U_{1}\right|>323208 \cdot t^{7 / 2} Y^{8}=a_{1} t^{7 / 2} Y^{8}, \quad\left|U_{2}\right|>122382 \cdot t^{4} Y^{8}=a_{2} t^{4} Y^{8}, \\
\left|U_{3}\right|>72512 \cdot t^{4} Y^{8}=a_{3} t^{4} Y^{8}, \quad\left|U_{4}\right|>323434 \cdot t^{7 / 2} Y^{8}=a_{4} t^{7 / 2} Y^{8}, \\
\left|U_{5}\right|>68003 \cdot t^{4} Y^{8}=a_{5} t^{4} Y^{8} .
\end{gathered}
$$

Unfortunately the $U$ 's may change sign if we do not assume $t>4000$, so it is really necessary to distinguish between small $(\leq 4000)$ and large $t$. Note that 4000 is not the "correct" value for the distinction, but it is the next nice round number. 
Our next aim is to compute lower bounds for $n_{1}$ and $n_{2}$. Let us note that $\beta_{i}+\bar{\beta}_{i}=1$ and $\beta_{i}, \bar{\beta}_{i}>0$, hence $\beta_{i} \bar{\beta}_{i} \leq 1 / 4$. Since $\left|U_{i}\right|=\beta_{i} \epsilon_{i}^{n_{i}}+\bar{\beta}_{i} \epsilon_{i}^{-n_{i}}$ we obtain

$$
\epsilon_{i}^{n_{i}}=\frac{\left|U_{i}\right|+\sqrt{\left|U_{i}\right|^{2}-4 \beta_{i} \bar{\beta}_{i}}}{2 \beta_{i}} \geq \frac{2\left|U_{i}\right|-1}{2 \beta_{i}}
$$

for $i=1,2,3$. Hence

$$
n_{1} \geq \frac{7 / 2 \log t-\log \beta_{1}+\log \left(a_{1}-1 / 2\right)}{\log \epsilon_{1}} \geq c_{n_{1}} \log t
$$

and

$$
n_{i} \geq \frac{4 \log t-\log \beta_{i}+\log \left(a_{i}-1 / 2\right)}{\log \epsilon_{i}} \geq c_{n_{i}} \log t,
$$

provided $i=2,3$. Note that $\sqrt{x^{2}-1} \geq x-1$ for all $x \geq 1$. Since for $i=1,2,3$

$$
-\log \beta_{i}+\log \left(a_{i}-1 / 2\right)>0,
$$

we may choose

$$
c_{n_{1}}=\frac{7}{2 \log (3+2 \sqrt{2})}, \quad c_{n_{2}}=\frac{4}{\log (2+\sqrt{3})}, \quad c_{n_{3}}=\frac{4}{\log \eta} .
$$

Now, let us find bounds for $n_{1} / n_{2}$ and $n_{3} / n_{2}$. Because of (8) we have

$$
\left|\alpha_{1,2}\right|+\frac{\theta c_{1,2}}{U_{2}^{2}}=\frac{\left|U_{1}\right|}{\left|U_{2}\right|}=\frac{\beta_{1}}{\beta_{2}} \frac{\epsilon_{1}^{n_{1}}}{\epsilon_{2}^{n_{2}}} \frac{1+\beta_{1} / \bar{\beta}_{1} \cdot \epsilon_{1}^{-2 n_{1}}}{1+\beta_{2} / \bar{\beta}_{2} \cdot \epsilon_{2}^{-2 n_{2}}}
$$

for some $|\theta| \leq 1$. Further, we find

$$
\frac{\epsilon_{1}^{n_{1}}}{\epsilon_{2}^{n_{2}}}=\frac{\beta_{2}}{\beta_{1}}\left|\alpha_{1,2}\right|\left(1+\theta c_{2}\right)
$$

with $|\theta| \leq 1$. In particular we have $c_{2}=\frac{3.2148 \cdot 10^{6}}{t^{4}}$. Similarly we obtain

$$
\frac{\epsilon_{1}^{n_{3}}}{\epsilon_{2}^{n_{2}}}=\frac{\beta_{2}}{\beta_{3}}\left|\alpha_{3,2}\right|\left(1+\theta c_{2}\right)
$$

where in this case $c_{2}=\frac{3.0216 \cdot 10^{6}}{t^{4}}$. In particular we have

$$
\frac{3.6177}{\sqrt{t}}<\frac{\epsilon_{1}^{n_{1}}}{\epsilon_{2}^{n_{2}}}<\frac{7.1922}{\sqrt{t}}
$$

and

$$
0.80232<\frac{\epsilon_{3}^{n_{3}}}{\epsilon_{2}^{n_{2}}}<1.7836
$$

Taking logarithms in the first case we obtain

$$
n_{1} \log \epsilon_{1}-n_{2} \log \epsilon_{2}=\log \left(\frac{\beta_{2}}{\beta_{1}}\left|\alpha_{1,2}\right|\right)+2 \theta c_{2}
$$

for some $|\theta|<1$. Note that for $|1-x|<1 / 3$ we have $|\log (1+x)|<2|x|$. Further manipulations yield

$$
\frac{n_{1}}{n_{2}}=\frac{\log \epsilon_{2}}{\log \epsilon_{1}}+\frac{\log \left(\frac{\beta_{2}}{\beta_{1}}\left|\alpha_{1,2}\right|\right)+2 \theta c_{2}}{n_{2} \log \epsilon_{1}}=\frac{\log \epsilon_{2}}{\log \epsilon_{1}}+r
$$


and

$$
\frac{n_{3}}{n_{2}}=\frac{\log \epsilon_{2}}{\log \epsilon_{3}}+\frac{\log \left(\frac{\beta_{2}}{\beta_{1}}\left|\alpha_{1,2}\right|\right)+2 \theta c_{2}}{n_{2} \log \epsilon_{3}}=\frac{\log \epsilon_{2}}{\log \epsilon_{3}}+r,
$$

with $|r|<c_{3}$ and $c_{3}=0.09339$ in the first case and $c_{3}=\frac{1.67984}{\log t \log \eta}$ in the other case.

By (2) and the explicit computation of the constants in (8) we obtain

$$
\begin{aligned}
\left|U_{1}\left(1-\zeta_{1}-\zeta_{2}-\zeta_{3}\right)+U_{2}\left(\zeta_{1}+\alpha_{2,3} \zeta_{2}+\alpha_{2,4} \zeta_{3}-\alpha_{2,5}\right)\right| & \\
& <\frac{\left|c_{3,2} \zeta_{2}\right|+\left|c_{4,2} \zeta_{3}\right|+c_{5,2}}{\left|U_{2}\right|}=\frac{c_{4}}{\left|U_{2}\right|},
\end{aligned}
$$

where $c_{4}=\frac{2.3345 \cdot 10^{6}}{t^{4}}$. Because of Lemma 1 we now obtain

$$
\begin{array}{r}
\left|\beta_{1} \epsilon_{1}^{n_{1}}\left(1-\zeta_{1}-\zeta_{2}-\zeta_{3}\right)+\beta_{2} \epsilon_{2}^{n_{2}}\left(\zeta_{1}+\alpha_{2,3} \zeta_{2}+\alpha_{2,4} \zeta_{3}-\alpha_{2,5}\right)\right| \\
\leq \frac{\tilde{c}_{5}}{\left|\beta_{1} \epsilon_{1}^{n_{1}}+\bar{\beta}_{1} \epsilon_{1}^{-n_{1}}\right|}+\left|\bar{\beta}_{1} \epsilon_{1}^{-n_{1}}\left(1-\zeta_{1}-\zeta_{2}-\zeta_{3}\right)\right| \\
+\left|\bar{\beta}_{2} \epsilon_{2}^{-n_{2}}\left(\zeta_{1}+\alpha_{2,3} \zeta_{2}+\alpha_{2,4} \zeta_{3}-\alpha_{2,5}\right)\right| \\
\leq c_{5} \epsilon_{1}^{-n_{1}}+c_{6} \epsilon_{2}^{-n_{2}} \leq c_{7} \epsilon_{2}^{-n_{2}} .
\end{array}
$$

The last inequality is obtained by (15). Moreover, by computing the constants explicitly we obtain $c_{5}=0.48428, c_{6}=0.0091655$ and $c_{7}=0.13401 \sqrt{t}$. Further manipulations yield (10) and hence (11) with $c=c_{8}=0.039454 t$. In particular we obtain the following lemma:

Lemma 3. Assume $t>4000$. Then we have

$$
|\Lambda|=\left|n_{1} \log \epsilon_{1}-n_{2} \log \epsilon_{2}+\log \gamma\right|<c_{9} \epsilon_{2}^{-2 n_{2}}
$$

with $c_{9}<4.43996 t$ in all cases. For more details see Table 2 .

TABLE 2. Values of $c_{9}$ in the case $t>4000$.

\begin{tabular}{|c|c||c|c||c|c||c|c|}
\hline$j=1$ & $0.0395 t$ & $j=5$ & $0.0468 t$ & $j=9$ & $3.723 t$ & $j=13$ & $0.0648 t$ \\
\hline$j=2$ & $0.0199 t$ & $j=6$ & $0.0641 t$ & $j=10$ & $0.04295 t$ & $j=14$ & $0.000221 t$ \\
\hline$j=3$ & $0.0477 t$ & $j=7$ & $0.000265 t$ & $j=11$ & $0.00112 t$ & $j=15$ & $4.44 t$ \\
\hline$j=4$ & $0.000578 t$ & $j=8$ & $0.0212 t$ & $j=12$ & $3.807 t$ & $j=16$ & $3.75 t$ \\
\hline
\end{tabular}

Next we want to apply a result on lower bounds for linear forms in logarithms to $\Lambda$. In particular we want to apply a result due to Matveev (see [15]):

Theorem 3. Denote by $\alpha_{1}, \ldots, \alpha_{n}$ algebraic numbers, not 0 nor 1, by $\log \alpha_{1}, \ldots$, $\log \alpha_{n}$ determinations of their logarithms, by $D$ the degree over $\mathbb{Q}$ of the number field $K=\mathbb{Q}\left(\alpha_{1}, \ldots, \alpha_{n}\right)$, and by $b_{1}, \ldots, b_{n}$ rational integers. Furthermore, let $\kappa=1$ if $K$ is real and $\kappa=2$ otherwise. Choose

$$
A_{i} \geq \max \left\{D h\left(\alpha_{i}\right),\left|\log \alpha_{i}\right|\right\} \quad(1 \leq i \leq n),
$$

where $h(\alpha)$ denotes the absolute logarithmic Weil height of $\alpha$ and

$$
B=\max \left\{1, \max \left\{\left|b_{j}\right| A_{j} / A_{n}: 1 \leq j \leq n\right\}\right\} .
$$

Assume $b_{n} \neq 0$ and $\log \alpha_{1}, \ldots, \log \alpha_{n}$ are linearly independent over $\mathbb{Z}$. Then

$$
\log \left|b_{1} \log \alpha_{1}+\cdots+b_{n} \log \alpha_{n}\right| \geq-C(n) C_{0} W_{0} D^{2} \Omega,
$$


with

$$
\begin{gathered}
\Omega=A_{1} \cdots A_{n}, \\
C(n)=C(n, \kappa)=\frac{16}{n ! \kappa} e^{n}(2 n+1+2 \kappa)(n+2)(4(n+1))^{n+1}\left(\frac{1}{2} e n\right)^{\kappa}, \\
C_{0}=\log \left(e^{4.4 n+7} n^{5.5} D^{2} \log (e D)\right), \quad W_{0}=\log (1.5 e B D \log (e D)) .
\end{gathered}
$$

Since we know $\gamma$ explicitly, it is no problem to compute all its conjugates and also its height. Therefore we compute

$$
h(\gamma) \leq 4.01445+8 \log t \leq 8.48402 \log t \quad(t>4000) .
$$

In the other cases the same inequality (21) holds. Therefore we choose $\alpha_{1}=$ $\epsilon_{1}, \alpha_{2}=\epsilon_{2}$ and $\alpha_{3}=\gamma$; moreover we take $A_{1}=8 \log (3+\sqrt{2}), A_{2}=8 \log (2+\sqrt{3})$ and $A_{3}=135.7442349 \log t$. Of course $D=16, n=3$ and $\kappa=1$. By this choice of the $A_{i}$ we obtain

$$
B=\max \left\{\frac{c_{10} n_{2}}{\log t}, 1\right\},
$$

with $c_{10}=0.097479$, i.e. $B=1$ for $n_{2}<\frac{1}{c_{10}} \log t<10.2588 \log t$. Therefore let us assume $n_{2} \geq 10.2588 \log t$. Then we obtain by Matveev's theorem (Theorem 3 )

$$
-c_{11} \log t \log \left(\frac{n_{2} c_{12}}{\log t}\right)<\log |\Lambda|<\log c_{9}-2 n_{2} \log \epsilon_{2},
$$

where $c_{11}=1.101191 \cdot 10^{17}$ and $c_{12}=23.9914$. Now let us assume $n_{2}=\xi \log t$. Then we obtain the inequality

$$
c_{11} \log \left(\xi c_{12}\right)<2 \xi \log \epsilon_{2}-\frac{\log c_{9}}{\log t},
$$

which is valid only for $\xi<c_{13}=1.89232 \cdot 10^{18}$. After similar computations in all other cases we get:

Proposition 1. Assume $t>4000$. Then we have $0 \leq n_{2}<c_{13} \log t=1.89925$. $10^{18} \log t$ and $0 \leq n_{3}<\frac{c_{14} \log t}{\log \eta}=\frac{2.92085 \cdot 10^{18} \log t}{\log \eta}$ in all cases.

Note that the inequality for $n_{2}$ is obtained immediately. The inequality for $n_{3}$ follows from the bound for $n_{2}$ and (18).

\section{SMall $t$ : II}

In this section, we want to apply a method introduced by Baker and Davenport [4. This method yields new upper bounds for $n_{2}$ for every specific $t$. So it is possible to show that the only solutions to (1) are trivial, provided $t<10^{7}$.

First, let us recall that $\frac{n_{1}}{n_{2}}<\frac{\log \epsilon_{2}}{\log \epsilon_{1}}+c_{3}<1$, and therefore $n_{1}<n_{2}$. Now we may state a variant of a result due to Baker and Davenport [4]:

Lemma 4. Assume $\max \left\{n_{1}, n_{2}\right\}<N$ and let $\kappa>1$ be a real number. Also assume there exists a convergent $p / q$ to $\delta_{1}:=\frac{\log \epsilon_{1}}{\log \epsilon_{2}}$ with $q<\kappa N$ such that

$$
\left\|q \delta_{1}\right\|<\frac{1}{2 \kappa N} \quad \text { and } \quad\left\|q \delta_{2}\right\|>\frac{1}{\kappa}
$$


where $\delta_{2}:=\frac{\log \gamma}{\log \epsilon_{2}}$ and $\|\cdot\|$ denotes the distance to the nearest integer. Then we have

$$
\max \left\{n_{1}, n_{2}\right\} \leq \frac{\log \left(\frac{2 \kappa^{2} N c_{9}}{\log \epsilon_{2}}\right)}{2 \log \epsilon_{2}} .
$$

Proof. We consider equation (20), divide it by $\log \epsilon_{1}$ and multiply it by $q$. Then under our assumptions we obtain

$$
\frac{1}{2 \kappa}<\left\|q \delta_{2}\right\|-n_{1}\left\|q \delta_{1}\right\| \leq\left|n_{1}\left(q \frac{\log \epsilon_{1}}{\log \epsilon_{2}}-p\right)+q \frac{\log \gamma}{\log \epsilon_{2}}+n_{2} q+n_{1} p\right|<\frac{q c_{9} \epsilon_{2}^{-2 n_{2}}}{\log \epsilon_{2}} .
$$

Since $q<\kappa N$, this yields

$$
\frac{1}{2 \kappa}<\frac{N \kappa c_{9} \epsilon_{2}^{-2 n_{2}}}{\log \epsilon_{2}}
$$

Solving this inequality for $n_{2}$ we obtain the lemma.

Due to this lemma we use the following algorithm to solve Thue equation (1) for each admissible $t$ with $4001 \leq t \leq 10^{7}$. First, we make some precomputations by computing convergents $p / q$ to $\delta_{1}$ up to the 55 -th convergent. Moreover, we consider only those $q$ 's such that $\left\|q \delta_{1}\right\|<\left(2 \cdot \max \left\{c_{13}\right\} \cdot \log 10^{7}\right)^{-1}$ to ensure $\kappa>1$. Note that for every case $j$ we get an upper bound for $n_{2}$ of the form $n_{2}<c_{13} \log t$. The "worst" case is the maximum of the $c_{13}$ 's, and the "best possible" case is the minimum of the $c_{13}$ 's if $c_{13}$ is considered as a 16-tuple corresponding to the various cases. By this selection process only 15 of these $q$ 's are left, with the smallest being $q=q_{1}=59666063706602912133$. For each of these $q$ 's we compute

$$
\frac{1}{\left\|q \delta_{1}\right\| 2 \cdot \max \left\{c_{13}\right\} \cdot \log 10^{7}}
$$

which yields an upper bound for $\kappa$, e.g. for $q_{1}$ we obtain $\kappa<374.659$. But on the other hand $q<\kappa \min \left\{c_{13}\right\} \log 4000$; hence we have a lower bound for $\kappa$, e.g. for $q_{1}$ we obtain $\kappa>3.8016$. If upper and lower bounds provide no contradiction, we have found an admissible $q$ and a bound for $\kappa$. In particular, there are eleven $q$ 's left. For instance in the case $q_{1}$ we choose $\kappa=300$ in order to have a good chance that $\left\|q \delta_{2}\right\|>\frac{1}{\kappa}$. For the remaining $q$ 's we choose the respectively lower bounds for $\kappa$ (see Table 3 below).

TABle 3. Addmissable $q$ 's and the corresponding $\kappa$.

\begin{tabular}{|l|l|}
\hline$q$ & $\kappa$ \\
\hline \hline 59666063706602912133 & 300 \\
\hline 22952311696019982651958 & 1462.4 \\
\hline 91749580720373327695699 & 5845.8 \\
\hline 389950634577513293434754 & 24846 \\
\hline 9060614176003179076695041 & 577294 \\
\hline 44913120245438382090040451 & $2.862 \cdot 10^{6}$ \\
\hline 817496778593894056697423159 & $5.209 \cdot 10^{7}$ \\
\hline 38467261714158459046868928924 & $2.451 \cdot 10^{9}$ \\
\hline 154686543635227730244173138855 & $9.856 \cdot 10^{9}$ \\
\hline 1740019241701663491732773456329 & $1.109 \cdot 10^{11}$ \\
\hline 71186102366132975430799538570634 & $4.536 \cdot 10^{12}$ \\
\hline
\end{tabular}


We sequentially try these $q$ 's in order to apply Lemma 4. It is highly improbable that we need more than the first five $q$ 's to apply the lemma. Note that by a simple heuristic the probability that we need the 6 -th $q$ is less than $1.15 \cdot 10^{-18}$. Indeed, to test all cases up to $t=10^{7}$ we need only the first five $q$ 's. This happens only four times. However, by the lemma we have a new upper bound for $n_{2}$. Compairing this new upper bound with the lower bound (14), we obtain either a contradiction or a "small" range for $n_{2}$ and hence for $n_{1}$. If the latter case occurs we check whether these finitely many cases yield a solution to (20) and may therefore find all nontrivial solutions to (11). Indeed an implementation of this algorithm and a computer search shows that there are no nontrivial solutions. This algorithm was implemented in Mathematica and was executed on a usual PC (Dual Core 2.8 Ghz, 4 GB Memory) for the cases $4001 \leq t \leq 10^{7}$ in 16 days. Therefore we may assume $t>10^{7}$ for the rest of the paper.

\section{A Better Bound}

In this section, we recompute the bounds obtained from section 4, but this time we assume $t>10^{7}$. Doing so, we have for $j=1$ (see (17) and (18))

$$
\frac{n_{1}}{n_{2}}=\frac{\log \epsilon_{2}}{\log \epsilon_{1}}+\theta c_{3}
$$

respectively

$$
\frac{n_{3}}{n_{2}}=\frac{\log \epsilon_{2}}{\log \epsilon_{3}}+\theta c_{3}
$$

for some $|\theta| \leq 1$, and $c_{3}=0.093389$ in the first case and $c_{3}=\frac{0.05736}{\log t \log \eta}$ in the other case. Furthermore, we obtain (see (20)

$$
|\Lambda|=\left|n_{1} \log \epsilon_{1}-n_{2} \log \epsilon_{2}+\log \gamma\right|<c_{9} \epsilon_{2}^{-2 n_{2}},
$$

with $c_{9}<3.49956 t$ for all $j$ 's. For more details see Table 4 .

TABLE 4. Values of $c_{9}$ in the case $t>10^{7}$.

\begin{tabular}{|c|c||c|c||c|c|}
\hline$j=1$ & $0.0182633 t$ & $j=7$ & $0.0001034 t$ & $j=12$ & $3.489 t$ \\
\hline$j=2$ & $0.0179929 t$ & $j=8$ & $0.0180155 t$ & $j=13$ & $0.0199443 t$ \\
\hline$j=3$ & $0.0200056 t$ & $j=9$ & $3.48752 t$ & $j=14$ & $0.0001026 t$ \\
\hline$j=4$ & $0.0001055 t$ & $j=10$ & $0.0198862 t$ & $j=15$ & $3.49956 t$ \\
\hline$j=5$ & $0.0182196 t$ & $j=11$ & $0.0001043 t$ & $j=16$ & $3.48792 t$ \\
\hline$j=6$ & $0.0201448 t$ & & & & \\
\hline
\end{tabular}

Applying Matveev's theorem again (see [15] or Theorem 3) we obtain $n_{2}<$ $c_{13} \log t=1.839772 \cdot 10^{18} \log t$ and $0 \leq n_{3}<\frac{c_{14} \log t}{\log \eta}=\frac{2.61465 \cdot 10^{18} \log t}{\log \eta}$ in all cases. By this last statement we know $n_{3}=0$ if $\log \eta>2.61465 \cdot 10^{18} \log t$. We claim the following

Lemma 5. Suppose $n_{3}=0$. Then there exist only trivial solutions.

Proof. If $n_{3}=0$ we immediately obtain $U_{3}+A_{3} V_{3}= \pm 1$ and $V_{3}=0$. But, in the case of $j=1$, by inequalities (12) we know $V_{3}<-72541 t^{7 / 2}<-1$ for $t>4000$. A contradiction, unless $Y=0$, i.e. $(X, Y)$ is a trivial solution. The other cases run analogously. 
Immediately we have

Corollary 2. If $t \leq 10^{7}$ or $\log \eta_{t}>2.61465 \cdot 10^{18} \log t$, then Thue equation (1) has only trivial solutions.

\section{LiNEAR FORMS IN THREE LOGARITHMS}

In view of Theorem 1 we see that the bound established in the section above applies only for huge $t$. Therefore we want to lower this bound in view of the proof of Theorem 2. These lower bounds will be established by using lower bounds for linear forms in three logarithms, as they were established by Bugeaud, Mignotte and Siksek [8] (see also [5]). We use the following variant (see [5, Proposition 3.3] for the case $\alpha_{i} \in \mathbb{Q}$ )

Theorem 4. Let $\alpha_{i}$ with $i=1,2,3$ be multiplicatively independent algebraic numbers all real or all complex, and consider the linear form

$$
\Lambda=b_{1} \log \alpha_{1}+b_{2} \log \alpha_{2}+b_{3} \log \alpha_{3},
$$

where $b_{i}$ are rational integers with $\operatorname{gcd}\left(b_{1}, b_{2}, b_{3}\right)=1$ and where the determinations of the logarithms are arbitrary but such that all are real or all purely imaginary. Let $L \geq 5$ be an integer and $m \geq 1, \chi>0, \rho>1$ be real parameters. Moreover, let

$$
a_{i} \geq \rho\left|\log \alpha_{i}\right|-\log \left|\alpha_{i}\right|+2 d h(\alpha)
$$

$i=1,2,3$, and $a_{i} \geq 1$, where $d=\left[\mathbb{Q}\left(\alpha_{1}, \alpha_{2}, \alpha_{3}\right): \mathbb{Q}\right] /\left[\mathbb{R}\left(\alpha_{1}, \alpha_{2}, \alpha_{3}\right): \mathbb{R}\right]$ and $a=\min \left\{a_{1}, a_{2}, a_{3}\right\}$, with $m a_{1} a_{2} a_{3} \geq 2$. Let us put $K=\left\lfloor m L a_{1} a_{2} a_{3}\right\rfloor$. Now, we define

$$
\begin{gathered}
C_{1}=\max \left\{(\chi m L)^{2 / 3}, \sqrt{2 m L / a}\right\}, \quad C_{2}=\left(2 m^{2} L^{2}\right)^{1 / 3}, \quad C_{3}=\left(6 m^{2}\right)^{1 / 3} L, \\
R_{i}=\left\lfloor C_{i} a_{2} a_{3}\right\rfloor, \quad S_{i}=\left\lfloor C_{i} a_{1} a_{3}\right\rfloor, \quad T_{i}=\left\lfloor C_{i} a_{1} a_{2}\right\rfloor,
\end{gathered}
$$

with $i=1,2,3$. Choose rational integers $R, S, T$ with

$$
R \geq R_{1}+R_{2}+R_{3}+1, \quad S \geq S_{1}+S_{2}+S_{3}+1, \quad T \geq T_{1}+T_{2}+T_{3}+1 .
$$

Assume

$$
\begin{gathered}
\left(\frac{K L}{2}+\frac{L}{4}-1-\frac{2 K}{3 L}\right) \log \rho \geq( \\
(d+1) \log \left(K^{2} L\right)+g L\left(a_{1} R+a_{2} S+a_{3} T\right) \\
+d(K-1) \log b-2 \log (e / 2)
\end{gathered}
$$

where

with

$$
g=\frac{1}{4}-\frac{K^{2} L}{12 R S T}, \quad b=\left(b_{2} \eta_{0}\right)\left(b_{2} \zeta_{0}\right)\left(\prod_{k=1}^{K-1} k !\right)^{-\frac{4}{K(K-1)}},
$$

Then either

$$
\eta_{0}=\frac{R-1}{2}+\frac{(S-1) b_{1}}{2 b_{2}}, \quad \zeta_{0}=\frac{T-1}{2}+\frac{(S-1) b_{3}}{2 b_{2}} .
$$

$$
\Lambda^{\prime}>\rho^{-K L}
$$

where

$$
\Lambda^{\prime}=|\Lambda| \max \left\{\frac{L R e^{L R|\Lambda| /\left(2 b_{1}\right)}}{2\left|b_{1}\right|}, \frac{L S e^{L S|\Lambda| /\left(2 b_{2}\right)}}{2\left|b_{2}\right|}, \frac{L T e^{L T|\Lambda| /\left(2 b_{3}\right)}}{2\left|b_{3}\right|}\right\},
$$

or one of the following conditions $(\mathbf{C 1}),(\mathbf{C 2}),(\mathbf{C 3})$ or $(\mathbf{C 4})$ hold with

$$
M=\chi \sqrt{\left(R_{1}+1\right)\left(S_{1}+1\right)\left(T_{1}+1\right)} .
$$


(C1): $\left|b_{1}\right| \leq R_{1}$ and $\left|b_{2}\right| \leq S_{1}$ and $\left|b_{3}\right| \leq T_{1}$.

(C2): $\left|b_{1}\right| \leq R_{2}$ and $\left|b_{2}\right| \leq S_{2}$ and $\left|b_{3}\right| \leq T_{2}$.

(C3): There exist two nonzero rational integers $r_{1}$ and $s_{1}$ such that $r_{1} b_{2}=$ $s_{1} b_{1}$ with

$$
\left|r_{1}\right| \leq \frac{\left(R_{1}+1\right)\left(T_{1}+1\right)}{M-\max \left\{R_{1}, T_{1}\right\}}, \quad\left|s_{1}\right| \leq \frac{\left(S_{1}+1\right)\left(T_{1}+1\right)}{M-\max \left\{S_{1}, T_{1}\right\}} .
$$

(C4): There exist rational integers $r_{1}, s_{1}, t_{1}$ and $t_{2}$, with $r_{1} s_{1} \neq 0$ such that

$$
\begin{aligned}
& \quad\left(t_{1} b_{1}+r_{1} b_{3}\right) s_{1}=r_{1} b_{2} t_{2}, \quad \operatorname{gcd}\left(r_{1}, t_{1}\right)=\operatorname{gcd}\left(s_{1}, t_{2}\right)=1 \\
& \text { and } \\
& 0<\left|r_{1} s_{1}\right| \leq \delta \frac{\left(R_{1}+1\right)\left(S_{1}+1\right)}{M-\max \left\{R_{1}, S_{1}\right\}}, \quad\left|s_{1} t_{1}\right| \leq \delta \frac{\left(S_{1}+1\right)\left(T_{1}+1\right)}{M-\max \left\{S_{1}, T_{1}\right\}}, \\
& \left|r_{1} t_{2}\right| \leq \delta \frac{\left(R_{1}+1\right)\left(T_{1}+1\right)}{M-\max \left\{R_{1}, T_{1}\right\}},
\end{aligned}
$$

where $\delta=\operatorname{gcd}\left(r_{1}, s_{1}\right)$.

We want to apply this theorem to (20). Therefore we have to perform some computations. We choose $\alpha_{1}=\gamma, \alpha_{2}=\epsilon_{2}$ and $\alpha_{3}=\epsilon_{1}$, therefore $b_{1}=1, b_{2}=n_{2}$ and $b_{3}=n_{1}$. Moreover, we choose

$$
\begin{aligned}
& a_{1}=((\rho-1) 0.723933+263.429982) \log t>(\rho-1) \log \gamma+32 h(\gamma), \\
& a_{2}=(\rho+15) \log (2+\sqrt{3})=(\rho-1) \log \epsilon_{2}+32 h\left(\epsilon_{2}\right), \\
& a_{3}=(\rho+15) \log (3+2 \sqrt{2})=(\rho-1) \log \epsilon_{2}+32 h\left(\epsilon_{2}\right) .
\end{aligned}
$$

and so we have $a=\min \left\{a_{1}, a_{2}, a_{3}\right\}=a_{2}$. Let us choose real numbers $m$ and $l$, which will be fixed later, and put

$$
L=\lfloor l \log \log t\rfloor \text { and } K=\left\lfloor m L a_{1} a_{2} a_{3}\right\rfloor .
$$

Note that we will choose one $l$ for all $t$; hence we only obtain an estimation for $L$. In particular we have (note $t>10^{7}$ )

$$
l \log \log t \geq L>l \log \log t-1>\left(l-\frac{1}{\log \log \left(10^{7}\right)}\right) \log \log t .
$$

Similarly, we obtain $c_{K} \log t \log \log t<K<\overline{c_{K}} \log t \log \log t$, where $c_{K}$ and $\overline{c_{K}}$ are effective computable constants depending on $l, m, \rho$ and $\chi$. Similarly, we find upper and lower bounds for $C_{1}, C_{2}, C_{3}, R_{i}, S_{i}, T_{i}, R, S, T$ and $g$ according to Theorem 4 . Later, when we have fixed $l, m, \chi$ and $\rho$, we will give exact estimates for these constants. Now we only want to give asymptotic expressions in order to know what we can expect from Theorem 4. In particular, we have

$$
\begin{gathered}
C_{1} \asymp C_{2} \asymp(\log \log t)^{2 / 3}, \quad C_{3} \asymp \log \log t, \\
R_{1} \asymp R_{2} \asymp(\log \log t)^{2 / 3}, \quad R=R_{1}+R_{2}+R_{3}+1 \asymp R_{3} \asymp \log \log t, \\
S_{1} \asymp S_{2} \asymp T_{1} \asymp T_{2} \asymp \log t(\log \log t)^{2 / 3}, \\
T=T_{1}+T_{2}+T_{3}+1 \asymp T_{3} \asymp S=S_{1}+S_{2}+S_{3}+1 \asymp S_{3} \asymp \log t \log \log t, \\
g \asymp 1 .
\end{gathered}
$$

The next quantity that we need is $b$. Therefore we have to compute the quantity $\left(\prod_{k=1}^{K-1} k !\right)^{-4 / K(K-1)}$. In partuicular we prove: 


\section{Lemma 6.}

$$
\log \left(\left(\prod_{k=1}^{K-1} k !\right)^{-\frac{4}{K(K-1)}}\right)=-2 \frac{(K-1) \log (K-1)}{K}+3 \frac{K-1}{K}+r,
$$

with

$$
|r| \leq \frac{2+4 \log K-1}{K}+\frac{7+4 \log K-1}{K(K-1)} .
$$

Proof. The proof is straightforward using Euler-Maclaurin's sum formula. Therefore we give only a rough overview. First, note that we have

$$
\log \left(\prod_{k=1}^{N} k !\right)=\sum_{k=1}^{N}(N+1-k) \log k=(N+1) \sum_{k=1}^{N} \log k-\sum_{k=1}^{N} k \log k .
$$

By Euler-Maclaurin's sum formula we obtain

$$
\sum_{k=1}^{N} \log k=\int_{1}^{N} \log x d x+\frac{\log N-\log 1}{2}+r_{1}
$$

and

$$
\sum_{k=1}^{N} k \log k=\int_{1}^{N} x \log x d x+\frac{N \log N-1 \log 1}{2}+r_{2}
$$

with

$$
r_{1}=\int_{1}^{N}(x-\lfloor x\rfloor-1 / 2) / x d x
$$

and

$$
r_{2}=\int_{1}^{N}(x-\lfloor x\rfloor-1 / 2)(\log x+1) d x .
$$

Put $N=K-1$, and after some straightforward computations one obtains the lemma.

Next, we have to compute $\log b$. By section 6 we know $b_{2}<c_{13} \log t=1.839772$. $10^{18} \log t$. Moreover, $b_{3} / b_{2}<\log \epsilon_{2} / \log \epsilon_{1}+c_{3}<c_{15}$ with $c_{15}=0.8823$, provided $t>10^{7}$. Therefore we obtain

$$
\begin{gathered}
\eta_{0}=\frac{R-1}{2}+\frac{(S-1) b_{1}}{2 b_{2}} \asymp \log \log t, \\
\zeta_{0}=\frac{T-1}{2}+\frac{(S-1) b_{3}}{2 b_{2}} \asymp \log t \log \log t .
\end{gathered}
$$


The result for $\eta_{0}$ is true, since by conditions $(\mathbf{C 1})$ and $(\mathbf{C 2})$ we may assume $b_{2}>$ $S_{1}, S_{2} \asymp \log t(\log \log t)^{2 / 3}$. Therefore we have

$$
\log b \asymp \log \left((\log t)^{3}(\log \log t)^{2}\right)-2 \log K \asymp \log \left(\frac{(\log t)^{3}(\log \log t)^{2}}{K^{2}}\right) \asymp \log \log t
$$

Now, let us consider inequality (26). Both the left and right hand sides are of asymptotic order $\log t(\log \log t)^{2}$. Hence we get an inequality in $m, l, \chi$ and $\rho$. For fixed values for $m, l$ and $\chi$, we get an inequality in $\rho$. If it is fulfilled for some $\rho>1$, we have found admissible parameters, and we get a lower bound for $\Lambda^{\prime}$ and also for $\Lambda$, hence a new upper bound for $b_{2}=n_{2}$. In particular, we have

$$
\begin{aligned}
-K L \log \rho<\log \left|\Lambda^{\prime}\right|=\log |\Lambda| & +\log \left(\max \left\{\frac{L R}{2 b_{1}}, \frac{L S}{2 b_{2}}, \frac{L T}{2 b_{3}}\right\}\right) \\
& +|\Lambda| \max \left\{\frac{L R}{2 b_{1}}, \frac{L S}{2 b_{2}}, \frac{L T}{2 b_{3}}\right\}
\end{aligned}
$$

hence

$$
-K L \log \rho-\log (L \max \{R, S, T\})<\log |\Lambda|+|\Lambda| \max \left\{\frac{L R}{2 b_{1}}, \frac{L S}{2 b_{2}}, \frac{L T}{2 b_{3}}\right\}-\log 2
$$

Let us assume $|\Lambda|<\frac{\rho^{-K L}}{L \max \{R, S, T\}}$. Then we obtain from the inequality above

$$
-K L \log \rho-\log (L \max \{R, S, T\})<\log |\Lambda|+\frac{1}{2}-\log 2<\log |\Lambda|
$$

a contradiction. Hence we have

$$
\log |\Lambda|>-K L \log \rho-\log (L \max \{R, S, T\}) \asymp \log t(\log \log t)^{2} .
$$

Note that the new lower bound for $n_{2}$ is asymptotically worse than the one obtained by Matveev's theorem, but the constants will be much smaller. Moreover, we have to be careful in our choice so that also the bounds coming from $(\mathbf{C 3})$ and $(\mathbf{C 4})$ stay significantly small. The bound obtained for (C3) turns into

$$
b_{2} \leq \frac{\left(S_{1}+1\right)\left(T_{1}+1\right)}{M-\max \left\{S_{1}, T_{1}\right\}}
$$

and condition (C4) turns into a linear form in two logarithms which will be discussed below. Hence $(\mathbf{C 3})$ and $(\mathbf{C 4})$ also yield bounds for $n_{2}$. With respect to these bounds we make the following choice:

$$
l=1461, \quad m=2, \quad \chi=1.999999, \quad \rho=18.394028 .
$$


With this choice we obtain:

$$
\begin{gathered}
1460.64 \log \log t<L<1461 \log \log t ; \\
2.087452 \cdot 10^{9} \log t \log \log t<K<2.087967 \cdot 10^{9} \log t \log \log t ; \\
324.3909(\log \log t)^{2 / 3}<C_{1}<324.4442(\log \log t)^{2 / 3} ; \\
257.4693(\log \log t)^{2 / 3}<C_{2}<257.5117(\log \log t)^{2 / 3} ; \\
4213.215 \log \log t<C_{3}<4214.254 \log \log t ; \\
839784.6(\log \log t)^{2 / 3}<R_{1}<839923.1(\log \log t)^{2 / 3} ; \\
666537.6(\log \log t)^{2 / 3}<R_{2}<666647.7(\log \log t)^{2 / 3} ; \\
10907197 \log \log t<R_{3}<10909885 \log \log t ; \\
11978485 \log \log t<R<11981349 \log \log t ; \\
5270736.9 \log t(\log \log t)^{2 / 3}<S_{1}<5271602.4 \log t(\log \log t)^{2 / 3} ; \\
4183388.1 \log t(\log \log t)^{2 / 3}<S_{2}<4184075 \log t(\log \log t)^{2 / 3} ; \\
68456759 \log t \log \log t<S_{3}<68473619 \log t \log \log t ; \\
75180476 \log t \log \log t<S<75198440 \log t \log \log t ; \\
3937795.9 \log t(\log \log t)^{2 / 3}<T_{1}<3938442.5 \log t(\log \log t)^{2 / 3} ; \\
3125431.7 \log t(\log \log t)^{2 / 3}<T_{2}<3125431.8 \log t(\log \log t)^{2 / 3} ; \\
51144413 \log t \log \log t<T_{3}<51157009 \log t \log \log t ; \\
56167738 \log t \log \log t<T<56181159 \log t \log \log t ; \\
0.239506<g
\end{gathered}
$$

In order to compute $b$ we have to compute the quantities $\eta_{0}$ and $\zeta_{0}$. Due to the conditions (C1) and (C2) and the computations made above, we may assume $b_{2}>S_{2}$. Therefore we obtain

$$
\eta_{0}<5990678.6799 \log \log t, \quad \zeta_{0}<61264333.1066 \log t \log \log t .
$$

By Lemma 6 and the computations above we obtain

$$
\log \left(\left(\prod_{k=1}^{K-1} k !\right)^{-\frac{4}{K(K-1)}}\right)<-17.0950171382 \log \log t .
$$

Hence, using the upper bounds for $n_{2}$ we get

$$
\log b<28.9611340689 \log \log t .
$$

With this choice inequality (26) is fulfilled. Therefore we have by the discussion above

$$
\log |\Lambda|>-K L \log \rho-\log (L \max \{R, S, T\})>-8.8831931 \cdot 10^{12} \log t(\log \log t)^{2},
$$

and hence

$$
n_{2}<3.372618 \cdot 10^{12} \log t(\log \log t)^{2}
$$

or one of the conditions $(\mathbf{C 1}),(\mathbf{C 2}),(\mathbf{C 3})$ or $(\mathbf{C 4})$ holds. On the other hand conditions $(\mathbf{C 1})$ and $(\mathbf{C 2})$ imply $n_{2}<S_{1}<5271602.4 \log t(\log \log t)^{2 / 3}$, respectively 
$n_{2}<S_{2}<4184075 \log t(\log \log t)^{2 / 3}$. Since $b_{1}=1$, condition (C3) turns into $r_{1} b_{2}=s_{1}$, and by Theorem 4 we get

$$
n_{2}=b_{2} \leq\left|s_{1}\right| \leq \frac{\left(S_{1}+1\right)\left(T_{1}+1\right)}{M-\max \left\{S_{1}, T_{1}\right\}}<2487.01868 \log t(\log \log t)^{1 / 3} .
$$

Now we investigate condition (C4), i.e. there exist integers $r_{1}, s_{1}, t_{1}$ and $t_{2}$ with $r_{1} s_{1} \neq 0$ such that $\left(t_{1} b_{1}+r_{1} b_{3}\right) s_{1}=r_{1} b_{2} t_{2}$ and let $\delta=\operatorname{gcd}\left(r_{1}, s_{1}\right)$. We consider the linear form $\Lambda$ and multiply it by $r_{1} t_{2} / \delta$ and then obtain

$$
\begin{aligned}
\left|\frac{r_{1} t_{2}}{\delta} \Lambda\right| & =\left|\frac{b_{3} r_{1} t_{2}}{\delta} \log \epsilon_{1}-\frac{b_{2} r_{1} t_{2}}{\delta} \log \epsilon_{2}+\frac{r_{1} t_{2}}{\delta} \log \gamma\right| \\
& =\left|\frac{b_{3} r_{1} t_{2}}{\delta} \log \epsilon_{1}-\frac{b_{3} r_{1} s_{1}}{\delta} \log \epsilon_{2}-\frac{t_{1} s_{1} b_{1}}{\delta} \log \epsilon_{2}+\frac{r_{1} t_{2}}{\delta} \log \gamma\right| \\
& =\left|b_{3} \log \sigma_{1}+\log \sigma_{2}\right|
\end{aligned}
$$

with $\sigma_{1}=\epsilon_{1}^{r_{1} t_{2} / \delta} \epsilon_{2}^{-r_{1} s_{1} / \delta}$ and $\sigma_{2}=\gamma^{r_{1} t_{2} / \delta} \epsilon_{2}^{-t_{1} s_{1} / \delta}$. Hence we have to consider a linear form in two logarithms, which imposes an application of the following theorem (Corollary 2 in [13]):

Theorem 5. Let $\alpha_{1}$ and $\alpha_{2}$ be two positive, real, multiplicatively independent elements in a number field of degree $D$ over $\mathbb{Q}$. For $i=1$ and $i=2$, let $\log \alpha_{i}$ be any determination of the logarithm of $\alpha_{i}$, and let $A_{i}>1$ be a real number satisfying

$$
\log A_{i} \geq \max \left\{h\left(\alpha_{i}\right),\left|\log \alpha_{i}\right| / D, 1 / D\right\} .
$$

Further, let $b_{1}$ and $b_{2}$ be two positive integers. Define

$$
b^{\prime}=\frac{b_{1}}{D \log A_{2}}+\frac{b_{2}}{D \log A_{1}} \quad \text { and } \quad \log b=\max \left\{\log b^{\prime}+0.14,21 / D, \frac{1}{2}\right\} .
$$

Then

$$
\left|b_{2} \log \alpha_{2}-b_{1} \log \alpha_{1}\right| \geq \exp \left(-24.34 D^{4}(\log b)^{2} \log A_{1} \log A_{2}\right) .
$$

We make our choice for $A_{1}$ and $A_{2}$ as follows. First, we compute the bounds for $r_{1} t_{2}, r_{1} s_{1}$ and $s_{1} t_{1}$ given in Theorem 4

$$
\begin{aligned}
\frac{r_{1} t_{2}}{\delta} & \leq 396.21129162(\log \log t)^{1 / 3}, \\
\frac{r_{1} s_{1}}{\delta} & \leq 530.38875262(\log \log t)^{1 / 3}, \\
\frac{s_{1} t_{1}}{\delta} & \leq 2487.0186727 \log t(\log \log t)^{1 / 3} .
\end{aligned}
$$

These bounds now yield

$$
h\left(\sigma_{1}\right) \leq \frac{r_{1} t_{2}}{\delta} h\left(\epsilon_{1}\right)+\frac{r_{1} s_{1}}{\delta} h\left(\epsilon_{2}\right) \leq 698.46(\log \log t)^{1 / 3}=\log A_{1}
$$

and

$$
h\left(\sigma_{1}\right) \leq \frac{r_{1} t_{2}}{\delta} h(\gamma)+\frac{t_{1} s_{1}}{\delta} h\left(\epsilon_{2}\right) \leq 4899.335 \log t(\log \log t)^{1 / 3}=\log A_{2} .
$$

Therefore we find

$$
b^{\prime}=\leq \frac{1}{\log A_{1}}+\frac{c_{13} \log t}{\log A_{2}}=\frac{2.070725565 \cdot 10^{13}}{(\log \log t)^{1 / 3}} .
$$


Hence

$$
\begin{aligned}
\log b & =\max \left\{\log b^{\prime}+0.14, \frac{21}{16}, \frac{1}{2}\right\} \\
& \leq \log \left(\frac{2.070725565 \cdot 10^{13}}{\left(\log \log 10^{7}\right)^{1 / 3}}\right)+0.14<30.46069518,
\end{aligned}
$$

and therefore we have

$$
\log \left|\frac{r_{1} t_{2}}{\delta} \Lambda\right|>-5.064759008 \cdot 10^{15} \log t(\log \log t)^{2 / 3} .
$$

Compairing this lower bound with the upper bound (20) for $|\Lambda|$, we deduce $n_{2} \leq$ $1.9229009 \cdot 10^{15} \log t(\log \log t)^{2 / 3}$. By inequality (18), the bounds for $n_{2}$ found in this section and Lemma 5 together with Corollary 2, we immediately obtain Theorem 1 .

Note that the asymptotic expression of the upper bound for $n_{2}$ found in this section is larger than the bound found by Matveev's theorem (Theorem 31). However, for small $t$ the new bound is about $1 / 1000$ smaller than the old one. So we still have a significant improvement, at least for small $t$.

\section{QuAdRATIC FIELDS WITH LARGE DISCRIMINANT}

The aim of this section is to learn something about lower bounds for fundamental units of certain families of quadratic fields. These lower bounds will yield Corollary 1.

We start with the following proposition, which is a composition of various results due to Halter-Koch [9]:

Proposition 2. Let

$$
t=\left(l p^{k} q+c\right)+4 p^{k} q
$$

with integers $k, q \geq 2$ and $l, c \geq 1$. Let $p=1+l c$ and $l q+l q c+l c$ odd. Then $t \equiv 1$ $\bmod 4$, and we have

(1) If $q$ and $c$ are relative prime and $p$ and $q$ are multiplicatively independent, then

$$
\log \eta>\frac{\left(\log \frac{\sqrt{t}}{2}\right)^{3}}{6 \log p \log q} .
$$

(2) Assume $q$ and c are relative prime, $l=1, p=r s$ and $q=d p+r$ with $d \geq 1$ and $s, r \geq 2$. Further assume $r, s$ and $d s+1$ are multiplicatively independent. Then

$$
\log \eta>\frac{\left(\log \frac{\sqrt{t}}{2}\right)^{4}}{24 \log p \log q \log (s(d s+1))} .
$$

Proof. This proposition is a combination of [9. Theorem 1], in particular the first displayed formula of [9, page 177] and [9, Propositon 1.i and 1.ii]. Note that the choice of $p_{1}$ and $p_{2}$, respectively $p_{1}, p_{2}$ and $p_{3}$, can be found by reading the proof of [9, Proposition 1].

By the first displayed formula of [9, page 177] we are left to estimate

$$
S=\sum_{e_{1}, \ldots, e_{n}}\left(\log \frac{\sqrt{t}}{2}-\sum_{i=1}^{n} e_{i} \log p_{i}\right),
$$


where the sum runs over all nonnegative integers $e_{1}, \ldots, e_{n}$ with $\sum_{i=1}^{n} e_{i} \log p_{i} \leq$ $\log \frac{\sqrt{t}}{2}$. But this sum is an upper Darboux sum of the integral

$$
I=\int_{\mathcal{B}}\left(\frac{\sqrt{t}}{2}-\sum x_{i} \log p_{i}\right) d x_{1} \ldots d x_{n}
$$

where

$$
\mathcal{B}=\left\{\left(x_{1}, \ldots, x_{n}\right) \in \mathbb{R}^{n}: x_{i} \geq 0, \sum_{i=1}^{n} x_{i} \log p_{i} \leq \log \frac{\sqrt{t}}{2}\right\}
$$

Therefore we have

$$
\log \eta>S>I=\frac{\left(\log \frac{\sqrt{t}}{2}\right)^{n+1}}{(n+1) ! \log p_{1} \cdots \log p_{n}} .
$$

Now we specialice to two families. In particular in view of Proposition 2 we choose for the first family $c=l=1, p=2$ and $q=3$, and for the second family we make the choice $c=5, l=1$ and $p=6=r s$ with $r=3$ and $s=2$; hence for $d=3$ we obtain $q=d p+r=21$. Then we immediately obtain by Proposition 2 ,

Lemma 7. (1) Let $t=\left(3 \cdot 2^{k}+1\right)^{2}+12 \cdot 2^{k}$, then we have

$$
\log \eta>\frac{\left(\log \frac{\sqrt{t}}{2}\right)^{3}}{6 \log 2 \log 3}
$$

(2) Let $t=\left(21 \cdot 6^{k}+5\right)^{2}+84 \cdot 6^{k}$, then we have

$$
\log \eta>\frac{\left(\log \frac{\sqrt{t}}{2}\right)^{4}}{24 \log 6 \log 21 \log 14}
$$

We end this section by proving Corollary 11. First, we consider the family $t=$ $\left(3 \cdot 2^{k}+1\right)^{2}+12 \cdot 2^{k}$. By Lemmas 5 and 7 and inequality (18) we deduce that Thue equation (11) has only trivial solutions if

$$
\frac{9.98887706 \cdot 10^{16} \log t(\log \log t)^{2 / 3}}{(\log t-1.38629437)^{3}}<1
$$

provided $\log t<4.709 \cdot 10^{50}$. For the other $t$ 's we obtain similar inequalities which obviously hold. This inequality is fulfilled for all $t$ with $\log t>8.661040129689 \cdot 10^{8}$. Since $\log t>2 k \log 2+\log 9$, we find $k \geq 624761981$.

Now let us investigate the family $t=\left(21 \cdot 6^{k}+5\right)^{2}+84 \cdot 6^{k}$. In this case we have to consider the inequality

$$
\frac{1.51072166 \cdot 10^{19} \log t(\log \log t)^{2 / 3}}{(\log t-1.38629437)^{4}}<1 .
$$

This inequality is fulfilled for all $t$ with $\log t>4.53416732171 \cdot 10^{6}$, and since $\log t>$ $2 k \log 6+\log 441$ we have $k \geq 1265282$. This completes the proof of Corollary 1 


\section{Final computations}

This section is devoted to the proof of Theorem 2 for $k \leq 1265281$. For this purpose we again use the method of Baker-Davenport described in section 5 . In contrast to section 5 , here we cannot compute $\delta_{2}$ for large $k$. Note that for $k \rightarrow \infty$ we have $\log \gamma \sim k \log 6$. For our needs it is sufficient to compute only an asymptotic expansion of $\log \gamma$. In order to make error terms "exact", we use following notation: For two functions $g(t)$ and $h(t)$ we write $g(t)=L(h(t))$ if $|g(t)| \leq h(t)$ for all $t$. We use this notation in the middle of an expression in the same way as it is usually done with the $O$-notation.

Let us note that $\gamma$ is a rational function in the $\sqrt{t}$ and has coefficients in $\mathbb{Q}(\sqrt{2}, \sqrt{3}, \sqrt{5})$. By estimating lower terms we find (in the case of $j=1$ ) that

$$
\begin{aligned}
\log \gamma & =\log \left(\frac{B_{1} t^{8}+L\left(1.342 \cdot 10^{6} t^{15 / 2}\right)}{B_{2} t^{15 / 2}+L\left(5.938 \cdot 10^{6} t^{7}\right)}\right) \\
& =\log \left(B_{1}\right)-\log \left(B_{2}\right)+\frac{\log t}{2}+L\left(92.291 t^{-1 / 2}\right),
\end{aligned}
$$

where

$$
\begin{gathered}
B_{1}=13389+7728 \sqrt{3}+5985 \sqrt{5}+3456 \sqrt{15}, \\
B_{2}=49710 \sqrt{2}+28700 \sqrt{6}+22302 \sqrt{10}+12876 \sqrt{30},
\end{gathered}
$$

and we use $\log (g+L(h))=\log (g)+L(2 h / g)$, provided $g>2|h|$. Also note that

$$
\begin{aligned}
\log t & =\log \left(6^{k}\left(441+294 \cdot 6^{-k}+25 \cdot 6^{-2 k}\right)\right) \\
& =k \log 6+\log 441+L\left(1.3339 \cdot 6^{-2 k}\right) .
\end{aligned}
$$

So, in practice we are able to compute $\log \gamma$ and hence $\delta_{2}$ for each $k$.

Next, we cannot precompute the values for $q$ and $\kappa$ for all $k$ because the lower and upper bounds would yield contradictions. So we compute the first sixty convergents to $\delta_{1}$ similar to section [5. But now we consider for each $k$ with $3 \leq k \leq 1265281$ (for $k=1,2$ we have $t<10^{7}$ ) only those $q$ 's such that

$$
\left\|q \delta_{1}\right\|<\left(2 \cdot 1.9229009 \cdot 10^{15} \log t(\log \log t)^{2 / 3}\right)^{-1} .
$$

We consider those $q$ 's to ensure $\kappa>1$. In order to find them we use formula (27). But, on the other hand, we have

$$
q<\kappa \cdot 1.9229009 \cdot 10^{15} \log t(\log \log t)^{2 / 3},
$$

hence we have a lower bound for $\kappa$. Now for a certain $k$ we consider only those $q$ 's such that the lower bound is smaller than the upper bound and the lower bound is larger than 300. We make this additional assumption to ensure that the first $q$ in our list is applicable with a high probability in the sense of Lemma 4. Note that this lemma now yields a new lower bound for $n_{2}$ which is usually rather small. Indeed, by computing the lower bounds for all $k \leq 1265281$, it never happens that the lower bound $n_{2} \geq c_{n_{2}} \log t=\frac{4 \log t}{\log (2+\sqrt{3})}$ (see equation (14)) does not exceed the upper bound, as found by the method of Baker and Davenport, i.e. there are no nontrivial solutions for $k \geq 3$. Running an implementation in Mathematica took about two hours on a common work station (Dual Core $2.8 \mathrm{Ghz}, 4$ GB Memory) to check all cases. 


\section{REFERENCES}

[1] A. Baker. Linear forms in the logarithms of algebraic numbers. I, II, III. Mathematika 13:204216, 1966; ibid. 14:102-107, 1967; ibid., 14:220-228, 1967. MR0220680 (36:3732)

[2] A. Baker. Contributions to the theory of Diophantine equations. I. On the representation of integers by binary forms. Philos. Trans. Roy. Soc. London Ser. A, 263:173-191, 1967/1968. MR0228424 (37:4005)

[3] A. Baker. Linear forms in the logarithms of algebraic numbers. IV. Mathematika, 15:204-216, 1968. MR0258756 (41:3402)

[4] A. Baker and H. Davenport. The equations $3 x^{2}-2=y^{2}$ and $8 x^{2}-7=z^{2}$. Quart. J. Math. Oxford Ser. (2), 20:129-137, 1969. MR0248079 (40:1333)

[5] M. A. Bennett, K. Győry, M. Mignotte, and Á. Pintér. Binomial Thue equations and polynomial powers. Compos. Math., 142(5):1103-1121, 2006. MR.2264658(2007h:11044)

[6] Y. Bilu and G. Hanrot. Solving Thue equations of high degree. J. Number Theory, 60(2):373392, 1996. MR.1412969 (97k:11040)

[7] Y. Bilu and G. Hanrot. Thue equations with composite fields. Acta Arith., 88(4):311-326, 1999. MR1690372 (2000c:11047)

[8] Y. Bugeaud, M. Mignotte, and S. Siksek. Classical and modular approaches to exponential Diophantine equations. II. The Lebesgue-Nagell equation. Compos. Math., 142(1):31-62, 2006. MR2196761 (2007f:11032)

[9] F. Halter-Koch. Reell-quadratische Zahlkörper mit großer Grundeinheit. Abh. Math. Sem. Univ. Hamburg, 59:171-181, 1989. MR1049894 (91h:11116)

[10] C. Heuberger. On a family of quintic Thue equations. J. Symbolic Comput., 26(2):173-185, 1998. MR1635238 (99d:11134)

[11] C. Heuberger, A. Togbé, and V. Ziegler. Automatic solution of families of Thue equations and an example of degree 8. J. Symbolic Computation, 38:1145-1163, 2004. MR2093887 (2005h:11070)

[12] S. Lang. Algebraic number theory, volume 110 of Graduate Texts in Mathematics. SpringerVerlag, New York, second edition, 1994. MR.1282723 (95f:11085)

[13] M. Laurent, M. Mignotte, and Y. Nesterenko. Formes linéaires en deux logarithmes et déterminants d'interpolation. J. Number Theory, 55(2):285-321, 1995. MR 1366574 (96h:11073)

[14] G. Lettl, A. Pethö, and P. Voutier. On the arithmetic of simplest sextic fields and related Thue equations. In Number theory (Eger, 1996), pages 331-348. de Gruyter, Berlin, 1998. MR:1628852(99h:11031)

[15] E. M. Matveev. An explicit lower bound for a homogeneous rational linear form in logarithms of algebraic numbers. II. Izv. Ross. Akad. Nauk Ser. Mat., 64(6):125-180, 2000. MR1817252 (2002e:11091)

[16] A. Pethő. Complete solutions to families of quartic Thue equations. Math. Comp., 57(196):777-798, 1991. MR.1094956 (92e:11023)

[17] N. P. Smart. The algorithmic resolution of Diophantine equations, volume 41 of London Mathematical Society Student Texts. Cambridge University Press, Cambridge, 1998. MR:1689189 (2000c:11208)

[18] E. Thomas. Complete solutions to a family of cubic Diophantine equations. J. Number Theory, 34(2):235-250, 1990. MR:1042497 (91b:11027)

[19] A. Thue. Über Annäherungswerte algebraischer Zahlen. J. Reine und Angew. Math., 135:284305, 1909.

[20] N. Tzanakis. Explicit solution of a class of quartic Thue equations. Acta Arith., 64(3):271-283, 1993. MR.1225429(94e:11022)

[21] N. Tzanakis and B. M. M. de Weger. On the practical solution of the Thue equation. J. Number Theory, 31(2):99-132, 1989. MR987566 (90c:11018)

Institute for Analysis and Computational Number Theory, Graz University of TechNOlogy, Steyrergasse 30/IV, A-8010 Graz, Austria

E-mail address: ziegler@finanz.math.tugraz.at

Current address: Institute of Mathematics, University of Debrecen, H-4010 Debrecen, P.O.

Box 12, Hungary 